
ИММУНОТЕРАПИИ ОНКОЛОГИЧЕСКИХ ЗАБОЛЕВАНИЙ

\author{
() Е.А. Пигарова*, Л.К. Дзеранова, Н.Ф. Нуралиева, Г.А. Мельниченко
}

ФГБУ «Национальный медицинский исследовательский центр эндокринологии» Минздрава России, Москва

В этой лекции подробно рассматриваются эндокринопатии, связанные с применением ингибиторов иммунных конечных точек при онкологических заболеваниях, обсуждаются случаи, сроки и особенности клинических проявлений, предлагаются практические рекомендации для лечения пациентов.

КЛЮЧЕВЫЕ СЛОВА: ингибиторы иммунных конечных точек, ипилимумаб, ниволумаб, эндокринопатии, аутоиммунные заболевания, нежелательные явления, тиреоидит, гипофизит, надпочечниковая недостаточность, адреналит, гипотиреоз, меланома, рак легкого.

\title{
DIAGNOSIS AND TREATMENT OF ENDOCRINOLOGICAL COMPLICATIONS OF IMMUNOTHERAPY OF ONCOLOGICAL DISEASES
}

\author{
(c) Ekaterina A. Pigarova*, Larisa K. Dzeranova, N Nurana F. Nuralieva, Galina A. Melnichenko.
}

Endocrinology Research Centre, Moscow, Russia

In this lecture, we discuss in detail the endocrinopathies associated with the use of immune-checkpoint inhibitors in oncology. The cases, terms and features of clinical manifestations of endocrine-related immune reactions are discussed, and practical recommendations for the treatment of patients are proposed.

KEYWORDS: immune-checkpoint inhibitors, ipilimumab, nivolumab, endocrinopathies, autoimmune diseases, adverse effects, thyroiditis, pituitary, adrenal insufficiency, adrenalitis, hypothyroidism, melanoma, lung cancer, CTLA-4, PD-1.

\section{ВВЕДЕНИЕ}

Одними из величайших достижений современной онкологии являются разработка и внедрение в клиническую практику различных препаратов, создающих противораковый иммунитет. Привычные всем хирургия, облучение и химиотерапия действуют на саму опухоль, но, как известно, любой патологический процесс, а тем более бесконтрольное клеточное деление, не может происходить без влияния иммунитета.

Научной базой для создания этого нового подхода к лечению послужили открытия в области иммунологии у онкологических пациентов. Установлено, что при злокачественных опухолях в организме больных имеет место снижение иммунитета.

При онкопатологии имеются серьезные нарушения иммунного ответа и надзора за атипичными клетками и онкогенными вирусами. У каждого человека с течением времени образуются злокачественные клетки в любой ткани, но правильно функционирующий иммунитет распознает их, уничтожает и удаляет из организма. С возрастом иммунитет ослабляется, возможно, поэтому рак чаще диагностируется у пожилых людей.

Потенциальные раковые клетки, незрелые клетки различных тканей ежедневно и в больших количествах образуются у каждого индивидуума, что создает ежедневную теоретическую ситуацию для человека заболеть несколькими видами рака одновременно. Конечно, этого не случается по той причине, что постоянно срабатывает иммунная система и обезвреживает эти аномальные клетки, воспринимая их как чужеродные, и рак раз- вивается именно тогда, когда защитные механизмы этой системы дают сбой.

Основная цель иммунотерапии при раке - активизировать собственные защитные силы и сделать опухолевые элементы заметными для иммунных клеток и антител. Иммунотерапия принципиально отличается от привычных средств борьбы с онкологией, она активно внедряется в практику, препараты проходят масштабные клинические испытания, а врачи уже получают первые плоды своих многолетних исследований в виде излеченных больных.

Препараты ингибиторов иммунных контрольных точек (ИИКТ) уже являются частью терапевтического арсенала онкологов как эффективная терапия для неизлечимых неоплазий, таких как метастатическая меланома или рак легких. Ожидается, что их использование будет увеличиваться экспоненциально в ближайшем будущем, когда появятся дополнительные агенты, и их назначение будет распространено на различные типы опухолей.

К сожалению, применение этой группы препаратов связано с развитием гиперактивации аутоиммунитета с вовлечением различных органов и тканей, не стали исключением и эндокринная система и метаболические процессы, обусловленные ее нарушениями. Нежелательные явления со стороны эндокринной системы относятся к числу наиболее частых и сложных токсических осложнений, с которыми могут столкнуться онкологи, а некоторые могут быть опасными для жизни, если их не распознать вовремя. В то же время для эндокринологов эти нежелательные явления также могут представлять существенные трудности в дифференциальной диагностике и лечении пациентов. 
Таблица 1. Основные характеристики ингибиторов иммунных конечных точек для онкоиммунотерапии

\begin{tabular}{|c|c|c|}
\hline Агент & Функция & $\begin{array}{c}\text { Название } \\
\text { препарата (MHH) }\end{array}$ \\
\hline $\begin{array}{l}\text { Антитела } \\
\text { к CTLA-4 }\end{array}$ & $\begin{array}{c}\text { Цитотоксический Т-лимфоцит-ассоциированный антиген } 4 \text { (CTLA-4) является } \\
\text { ключевым рецептором, экспрессируемым на поверхности Т-лимфоцитов, } \\
\text { который проводит ингибирующий сигнал через свой лиганд B7-1/2, снижая } \\
\text { активацию Т-клеток. При блокировании CTLA-4 ингибирующий сигнал убирается } \\
\text { и Т-клеточная активация повышается }\end{array}$ & $\begin{array}{l}\text { Ипилимумаб } \\
\text { Тремелимумаб }\end{array}$ \\
\hline $\begin{array}{l}\text { Антитела } \\
\text { к PD1 }\end{array}$ & $\begin{array}{c}\text { Рецептор программируемой смерти } 1 \text { (PD1) является негативным регулятором } \\
\text { активации Т- и В-лимфоцитов и клеток натуральных киллеров, ограничивающим } \\
\text { аутоиммунное повреждение здоровых тканей }\end{array}$ & $\begin{array}{l}\text { Ниволумаб } \\
\text { Пембролизумаб } \\
\text { Пидилизумаб }\end{array}$ \\
\hline $\begin{array}{l}\text { Антитела } \\
\text { к PD-L1 }\end{array}$ & $\begin{array}{c}\text { Лиганд рецептора программируемой смерти } 1 \text { (PD-L1). Функция лиганда } \\
\text { аналогична рецептору PD1 }\end{array}$ & $\begin{array}{l}\text { Авелумаб } \\
\text { Атезолизумаб } \\
\text { Дурвалумаб }\end{array}$ \\
\hline
\end{tabular}

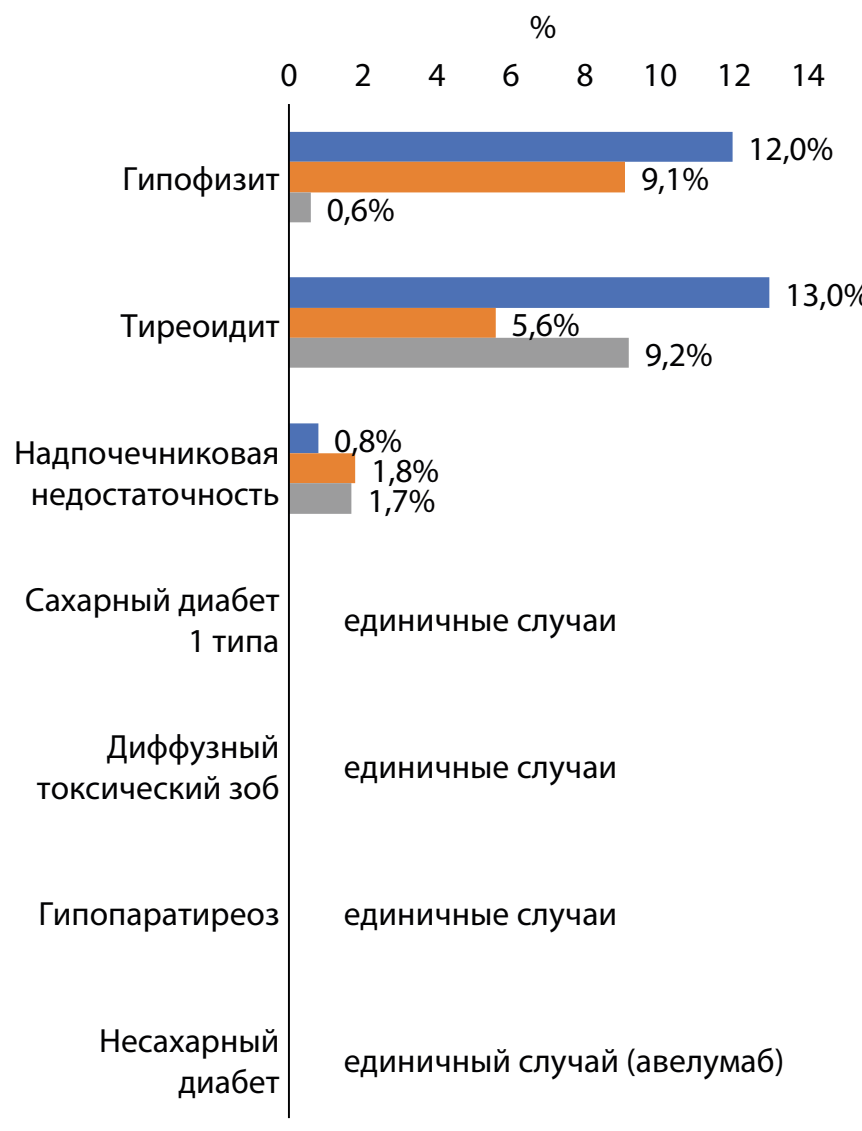

Рис. 1. Частота аутоиммуноопосредованных нежелательных явлений при применении ингибиторов иммунных конечных точек [1-5].

В этой лекции подробно рассматриваются эндокринопатии, связанные с применением ИИКТ, обсуждаются случаи, сроки и особенности клинических проявлений, предлагаются практические рекомендации для лечения пациентов.

\section{ПРЕПАРАТЫ ОНКОИММУНОТЕРАПИИ ИИКТ}

Препараты ИИКТ представляют собой человеческие антитела против различных мишеней, которые принимают активное участие в противоопухолевом иммунитете. Характеристика препаратов представлена в таблице 1. Разработаны схемы лечения как в виде монотерапии, так и комбинации препаратов с различным механизмом дей- ствия, поскольку экспериментально и клинически показано, что одновременное ингибирование CTLA-4 и PD1/ PD-L1 путей имеет синергический эффект [1].

\section{ЧАСТОТА ЭНДОКРИННЫХ НЕЖЕЛАТЕЛЬНЫХ ЯВЛЕНИЙ ПРИ ПРИМЕНЕНИИ ИИКТ}

Аутоиммуноопосредованные нежелательные явления (АОНЯ) могут появляться в период от начала терапии до трех месяцев после лечения и изначально казаться безвредными и связанными с лечением рака, а не с эндокринным расстройством. Согласно исследованиям, серьезные нежелательные реакции происходят в 20-30\% случаев при применении некоторых лекарственных средств в виде монотерапии и более чем в 50\%, когда эти препараты используются в комбинации [1].

При применении ИИКТ, наряду с аутоиммунным поражением кожи и желудочно-кишечного тракта, патология эндокринной системы является одной из наиболее распространенных. Частота различных эндокринологических АОНЯ представлена на рисунке 1.

В области эндокринологии мы наблюдаем несколько побочных эффектов ИИКТ. Эндокринные АОНЯ могут манифестировать с серьезных, жизнеугрожающих симптомов, как в случае гипофизита. В отличие от других АОНЯ, при эндокринных адекватное замещение гормональными препаратами быстро улучшает состояние, делая применение иммуносупрессивной терапии во многих случаях ненужным. Это позволяет пациентам продолжать противоопухолевое лечение и сохранять шансы на излечение от онкологического заболевания. Таким образом, выявление и правильная тактика лечения эндокринных АОНЯ крайне важны для оптимизации медицинской помощи и обеспечения максимального потенциала ИИКТ.

Частота и конкретная нозологическая форма АОНЯ зависят от препарата или комбинации используемых препаратов. При применении ипилимумаба эндокринные АОНЯ наблюдаются с частотой до $29 \%$, а самая частая форма гипофизит, аутоиммунное воспаление гипофиза, крайне редко встречается в общей популяции (около 1 случая на 9 млн населения). Реже возникают гипо- и гипертиреоз как следствие аутоиммунного процесса в щитовидной железе.

Научное обоснование такой высокой распространенности гипофизита при применении ИИКТ, как показыва- 
Таблица 2. Клинические и лабораторные проявления аутоиммунных заболеваний и их особенности при применении ингибиторов иммунных конечных точек

\begin{tabular}{|c|c|c|}
\hline $\begin{array}{l}\text { Заболевание/ } \\
\text { осложнение }\end{array}$ & $\begin{array}{c}\text { Симптомы/ } \\
\text { клинический } \\
\text { диагноз }\end{array}$ & $\begin{array}{c}\text { Диагностика/клинические } \\
\text { проявления }\end{array}$ \\
\hline & $\begin{array}{c}\text { Неврологические } \\
\text { проявления - } \\
\text { головная боль, } \\
\text { нарушение полей } \\
\text { зрения }\end{array}$ & $\begin{array}{c}\text { МРТ головного мозга } \\
\text { с контрастированием } \\
\text { Определение полей зрения } \\
\text { (периметрия) }\end{array}$ \\
\hline & \multicolumn{2}{|c|}{$\begin{array}{r}\text { Гормональные проявления - нарушение секреции гормо } \\
\text { гипофункцией периферических }\end{array}$} \\
\hline Гипофизит & $\begin{array}{c}\text { Вторичный } \\
\text { гипокортицизм } \\
\text { (вторичная } \\
\text { надпочечниковая } \\
\text { недостаточность) }\end{array}$ & $\begin{array}{c}\text { 1) Кортизол крови до 9:00: } \\
\text { - менее } 100 \text { нмоль/л подтверждает } \\
\text { гипокортицизм; } \\
\text { - 100-450 нмоль/л (нужна оценка } \\
\text { клинической картины); } \\
\text { - более } 450 \text { нмоль/л (исключает } \\
\text { гипокортицизм) } \\
\text { 2) АКТГ крови не повышен } \\
\text { относительно референсных } \\
\text { значений (дифференциальная } \\
\text { диагностика с первичной формой } \\
\text { надпочечниковой недостаточности) } \\
\text { В! Применение экзогенных } \\
\text { глюкокортикоидов (преднизолона, } \\
\text { метилпреднизолона, дексаметазона, } \\
\text { бетаметазона, триамцинолона) } \\
\text { приводит к подавлению АКтг } \\
\text { и снижению кортизола в крови в связи } \\
\text { с наличием обратной отрицательной } \\
\text { регуляторной связи на уровнях } \\
\text { гипоталамуса и гипофиза }\end{array}$ \\
\hline
\end{tabular}

ТТГ крови низкий или нормальный

Вторичный гипотиреоз

Свободный Т4 крови низкий
Остановить введение ИИКТ

Метилпреднизолон 1-2 мг/кг/сут

\section{Лечение}

- 


\begin{tabular}{cc}
\hline $\begin{array}{c}\text { Заболевание/ } \\
\text { осложнение }\end{array}$ & $\begin{array}{c}\text { Симптомы/ } \\
\text { клинический } \\
\text { диагноз }\end{array}$ \\
\hline
\end{tabular}

-недостаточность

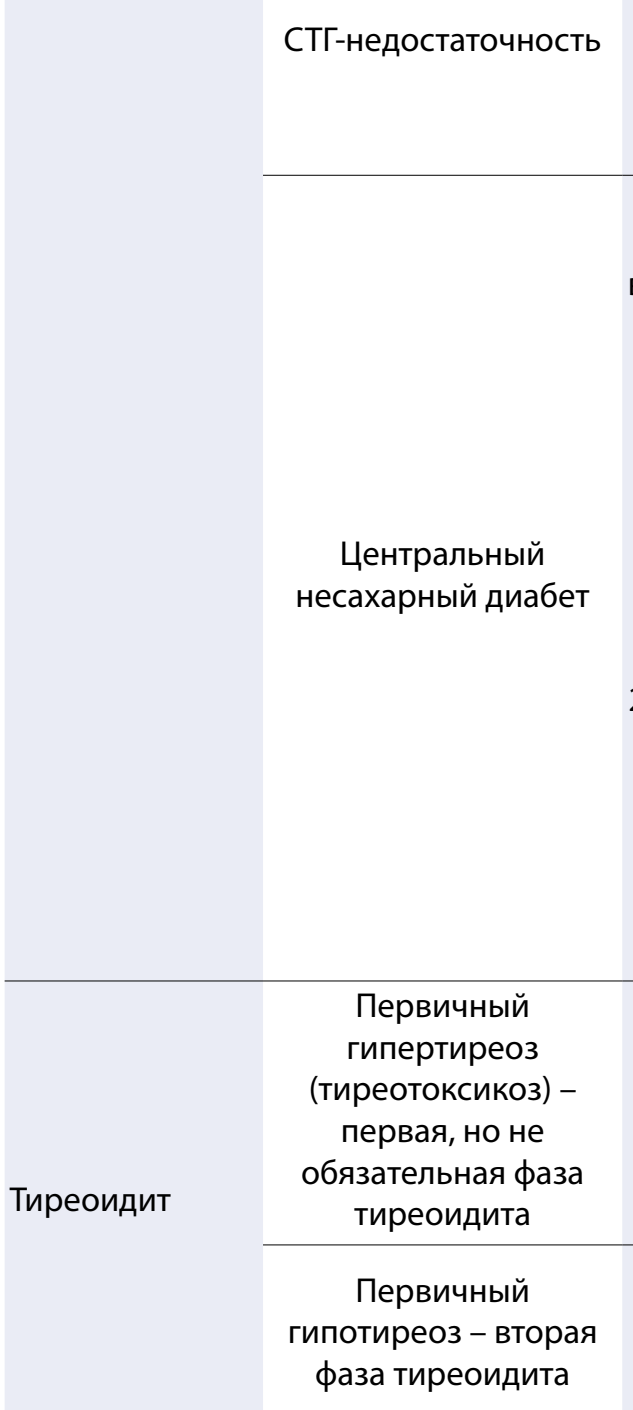

Адреналит

Первичная надпочечниковая

\section{Диагностика/клинические проявления}

Лечение

\section{Скрининг не показан}

1) ИФР-1 крови ниже референсных значений

2) СТГ на фоне стимуляционных проб

(с кломифеном, с инсулином) менее 10 нг/мл
Подозрением является появление жажды и полиурии более 3000 мл

в сутки, относительная плотность мочи менее 1005 г/л, осмоляльность мочи менее 300 мОсм/кг, без повышения глюкозы в крови или моче

\section{Диагностика}

1) В одновременно взятых анализах крови и мочи или при пробе с сухоедением:

осмоляльность крови более

295 мОсм/кг и/или натрий крови более 146 ммоль/л при осмоляльности мочи менее 300 мОсм/кг

2) Повышение осмоляльности мочи и исчезновение жалоб на полиурию и полидипсию на фоне приема десмопрессина

ТТГ крови низкий
Свободный Т4 и свободный Т3 крови высокие

\section{ТТГ крови высокий}

Свободный Т4 и свободный Т3 крови низкие или низко-нормальные

\section{АКТГ крови высокий}

Кортизол крови - низкий или низконормальный

Свободный кортизол в суточной моче низкий недостаточность
NB! Применение экзогенных глюкокортикоидов (преднизолона, метилпреднизолона, дексаметазона, бетаметазона, триамцинолона) приводит к подавлению АКТГ и снижению кортизола в крови в связи с наличием обратной отрицательной регуляторной связи на уровнях гипоталамуса и гипофиза

Лечение не показано

Десмопрессин:

- в таблетках подъязычных МЕЛТ по 60 мкг 2-3 раза в сутки; - в таблетках пероральных по 0,1 мг 2-3 раза в сутки

$N B$ ! Для каждой конкретной формы препарата подбирается минимальная дозировка, позволяющая контролировать избыточную жажду и полиурию

Симптоматическая терапия неселективными $\beta$-блокаторами (пропранолол 10-40 мг 3 раза в сутки)

L-тироксин в средней дозе 1,6 мкг на кг массы тела утром натощак за 30-40 мин до еды Цель - нормальные уровни ТТГ Назначение глюкокортикоидов и минералокортикоидов

Глюкокортикоиды: начало терапии по одной из схем с дальнейшей коррекцией доз препаратов по самочувствию:

гидрокортизон - по 15 мг утром, 10 мг в обед, 5 мг вечером;

кортизона ацетат - по 25 мг утром, 12,5 мг в обед, 5 мг вечером;

преднизолон - по 5 мг утром и 2,5 мг вечером

Минералокортикоиды: флудрокортизон 0,05-0,01 мг утром натощак Контроль дозы по стабильности АД, отсутствию/ наличию электролитных нарушений 


\begin{tabular}{|c|c|c|c|}
\hline $\begin{array}{c}\text { Заболевание/ } \\
\text { осложнение }\end{array}$ & $\begin{array}{c}\text { Симптомы/ } \\
\text { клинический } \\
\text { диагноз }\end{array}$ & $\begin{array}{c}\text { Диагностика/клинические } \\
\text { проявления }\end{array}$ & Лечение \\
\hline & $\begin{array}{l}\text { Аддисонический } \\
\text { криз (острая } \\
\text { надпочечниковая } \\
\text { недостаточность) }\end{array}$ & $\begin{array}{c}\text { В случае подозрения на острую } \\
\text { надпочечниковую недостаточность лечение } \\
\text { должно быть проведено незамедлительно } \\
\text { ввиду жизнеугрожающего характера } \\
\text { данного гормонального дефицита } \\
\text { До введения могут быть взяты анализы } \\
\text { крови на АКТГ и кортизол }\end{array}$ & $\begin{array}{c}\text { Препарат выбора: гидрокортизон } \\
\text { (Солу-Кортеф) в/в (предпочтительнее), } \\
\text { в/м, п/к } 100 \text { мг каждые 6-8 ч } \\
\text { Можно заменить: } \\
\text { преднизолон по 25-30 мг каждые } \\
8 \text { часов }\end{array}$ \\
\hline $\begin{array}{l}\text { Аутоиммунное } \\
\text { поражение } \\
\text { островков } \\
\text { Лангерганса }\end{array}$ & Сахарный диабет & $\begin{array}{c}\text { Повышение глюкозы крови более } \\
\text { 7,0 ммоль/л натощак и /или более } \\
\text { 11,1 ммоль/л через } 2 \text { ч после приема } 75 \text { г } \\
\text { глюкозы или в любое время тестирования } \\
\text { NB! Определение гликированного } \\
\text { гемоглобина для скрининга на } \\
\text { сахарный диабет НЕ РЕКОМЕНДУЕТСЯ, } \\
\text { т.к. при быстропрогрессирующем течении } \\
\text { возможны ложноотрицательные случаи }\end{array}$ & $\begin{array}{c}\text { Инсулинотерапия, инфузионная } \\
\text { терапия при наличии кетоацидоза, } \\
\text { электролитных нарушений }\end{array}$ \\
\hline $\begin{array}{l}\text { Аутоиммунное } \\
\text { поражение } \\
\text { вазопрессин- } \\
\text { секретирующих } \\
\text { клеток } \\
\text { гипоталамуса }\end{array}$ & $\begin{array}{c}\text { Центральный } \\
\text { несахарный диабет }\end{array}$ & $\begin{array}{c}\text { Подозрением является повышение } \\
\text { жажды и полиурии более } 3000 \text { мл в сутки, } \\
\text { относительная плотность мочи менее } \\
1005 \text { г/л, осмоляльность мочи менее } \\
300 \text { мОсм/кг, при отсутствии сахарного } \\
\text { диабета } \\
\text { Диагностика } \\
\text { 1) В одновременно взятых анализах крови } \\
\text { и мочи или при пробе с сухоедением: } \\
\text { осмоляльность крови более } 295 \text { мОсм/кг } \\
\text { и/или натрий крови более } 146 \text { ммоль/л } \\
\text { при осмоляльности мочи менее } \\
\text { 300 мОсм/кг; } \\
\text { 2) повышение осмоляльности мочи } \\
\text { и исчезновение жалоб на полиурию } \\
\text { и полидипсию на фоне приема } \\
\text { десмопрессина }\end{array}$ & $\begin{array}{c}\text { Десмопрессин: } \\
\text { - в таблетках подъязычных МЕЛт } \\
\text { по } 60 \text { мкг 2-3 раза в сутки; } \\
\text { - в таблетках пероральных по 0,1 мг } \\
\text { 2-3 раза в сутки } \\
\text { NB! Для каждой конкретной } \\
\text { формы препарата подбирается } \\
\text { минимальная дозировка, } \\
\text { позволяющая контролировать } \\
\text { избыточнуюжажду и полиурию }\end{array}$ \\
\hline $\begin{array}{l}\text { Аутоиммунное } \\
\text { поражение око- } \\
\text { лощитовидных } \\
\text { желез }\end{array}$ & Гипопаратиреоз & $\begin{array}{c}\text { Подозрением на заболевание является } \\
\text { подергивание и судороги в мышцах, } \\
\text { онемение в области губ и кистей рук } \\
\text { Кальций общий крови (или кальций } \\
\text { общий, скорректированный } \\
\text { на альбумин) - низкий } \\
\text { Паратгормон - низкий или низко- } \\
\text { нормальный при сниженном кальции } \\
\text { крови }\end{array}$ & 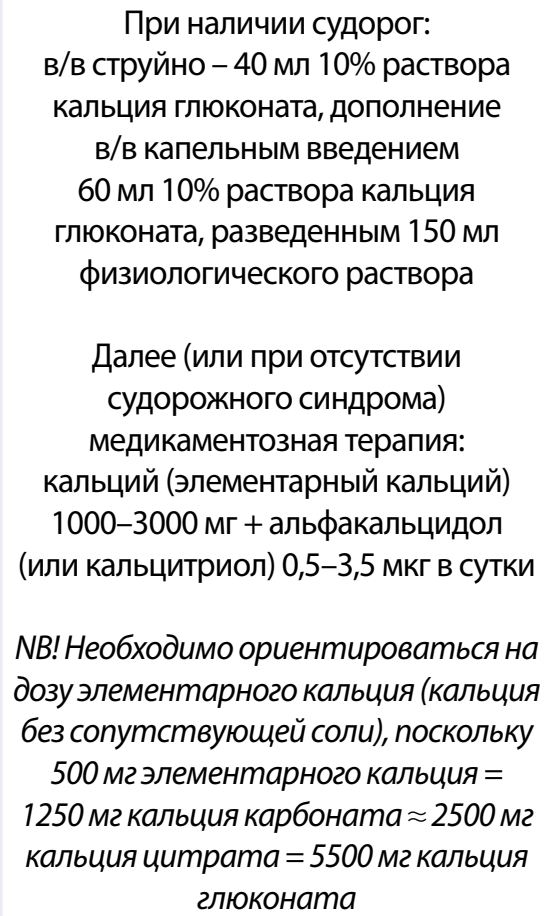 \\
\hline
\end{tabular}


Таблица 3. МРТ-отличия гипофизита от объемного образования гипофиза

\begin{tabular}{lcc}
\hline \multicolumn{1}{c}{ Критерии } & Гипофизит & Макроаденома \\
\hline Асимметрия объемного образования & Нет & Да \\
\hline Гомогенный сигнал до контрастирования & Да & Нет \\
\hline Нет изменений дна турецкого седла & Да & Нет \\
\hline Супраселлярное распространение & Да & Нет \\
\hline Утолщение ножки гипофиза & Нет & Да \\
\hline Отклонение ножки гипофиза в сторону & Да & Нет \\
\hline Гомогенное усиление при контрастировании & Часто & Редко \\
\hline $\begin{array}{l}\text { Потеря гиперинтенсивного сигнала от нейрогипофиза } \\
\text { (при его вовлечении в патологический процесс) }\end{array}$ & & \\
\hline
\end{tabular}

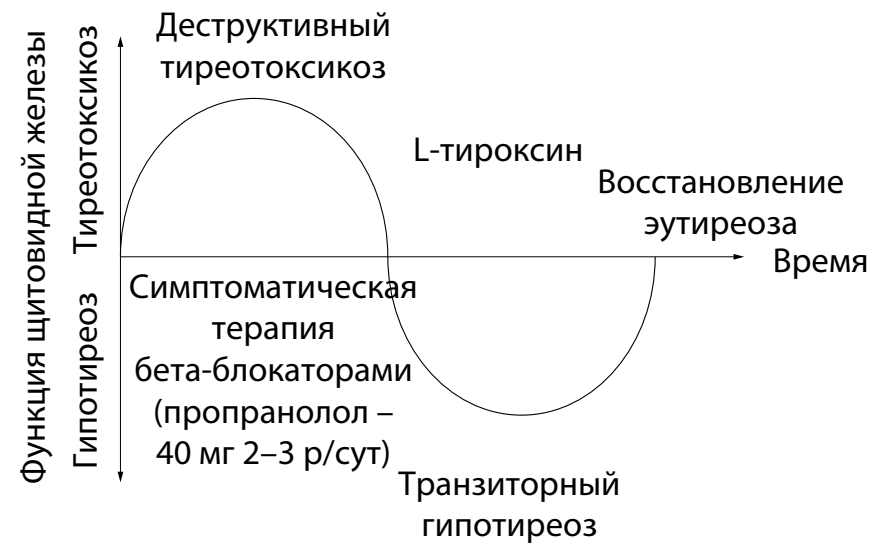

Рис. 2. Схематическое изображение фазного течения тиреоидита.

ют исследования, может быть в том, что клетки гипофиза (преимущественно пролактин (ПРЛ)- и тиреотропный гормон (ТТГ)-секретирующие) «эктопически» экспрессируют CTLA-4 рецепторы. При лечении ИИКТ образование комплексов IGG1 CTLA-4 (препарат) + рецептор CTLA-4 в гипофизе приводит к связыванию С1q с Fс фрагментом IGG CTLA-4 и к дальнейшей активации классического пути комплемента (продукция C3, C3d, C4d), что является II классом гиперчувствительности. Комплемент повреждает ткани гипофиза с активацией воспалительных лимфоцитов и синтезом аутоантител, усилением гипофизспецифического иммунного ответа с распространением на другие типы клеток [6, 7].

Эндокринологические АОНЯ обычно возникают после 6-й недели от начала применения ИИКТ, с медианой времени дебюта 7-20 нед. Эндокринопатии, как правило, не проходят бесследно, поскольку функция железы зачастую необратимо повреждается, хотя может быть полностью замещена экзогенными гормонами.

Другие эндокринологические АОНЯ, такие как первичная надпочечниковая недостаточность, сахарный диабет 1 типа, центральный несахарный диабет и гипопаратиреоз, также наблюдаются в связи с применением ИИКТ, но в редких или даже единичных случаях.

В исследованиях частота эндокринных АОНЯ значимо различается. Сравнительно более высокие цифры при более поздних работах отражают повышение осведомленности врачей об особенностях эндокринологических АОНЯ и улучшение их диагностики и лечения. Ведь такие неспецифические симптомы дебюта гипофизита, как головная боль, слабость, анорексия и тошнота, вместе с тем фактом, что гормональные нарушения часто встречаются у пациентов с распространенными формами рака, могут быть вовремя не распознаны. Диагностика также затруднена тем, что не всегда перед назначением ИИКТ проводится исходный гормональный скрининг (помимо оценки ТТГ), по неэндокринологическим показаниям применяются высокие дозы синтетических глюкокортикоидных препаратов, что может значимо влиять на интерпретацию результатов лабораторных анализов.

В настоящее время рекомендуется следующий объем лабораторного скрининга (по крови) на эндокринные АОНЯ с проведением исходно до начала лечения и до начала каждого цикла терапии препаратами ИИКТ:

- ТТГ и свободный Т4;

- кортизол (утром в 8:00-9:00);

- натрий, калий, кальций;

- глюкоза.

Такой объем обследования позволяет выявить субклинические или стертые формы эндокринологических АОНЯ, в том числе жизнеугрожающих, для своевременного начала лечения. Выявление отклонений на скрининге или наличие явных симптомов эндокринных АОНЯ, безусловно, требует расширения обследования для дальнейшей характеристики патологического процесса (табл. 2).

При подозрении на гипофизит на основании неврологических проявлений (головная боль, нарушение полей зрения, двоение в глазах и др.) или эндокринологических изменений при скрининге (снижение свободного Т4 без повышения ТТГ, гипогликемия, гипонатриемия, снижение кортизола крови) показаны МРТ головного мозга/гипофиза с контрастированием и дополнительно к скринингу определение гормонов, характеризующих гипофизарную функцию:

- адренокортикотропный гормон (АКТГ), кортизол (утром в 8-9:00);

- лютеинизирующий гормон (ЛГ), фолликулостимулирующий гормон (ФСГ), пролактин, у мужчин - дополнительно тестостерон.

МРТ головного мозга/гипофиза важна, но не обязательна для диагностики гипофизита, при наличии измененных соответствующих клинико-лабораторных показателей. Важной при проведении МРТ является дифференциальная диагностика гипофизита от метастазов в головной мозг/область гипофиза, для чего желательно сравнение с МРТ до лечения и/или использование критериев из таблицы 3 для вероятностного различения воспалительного увеличения гипофиза и объемного процесса в области 


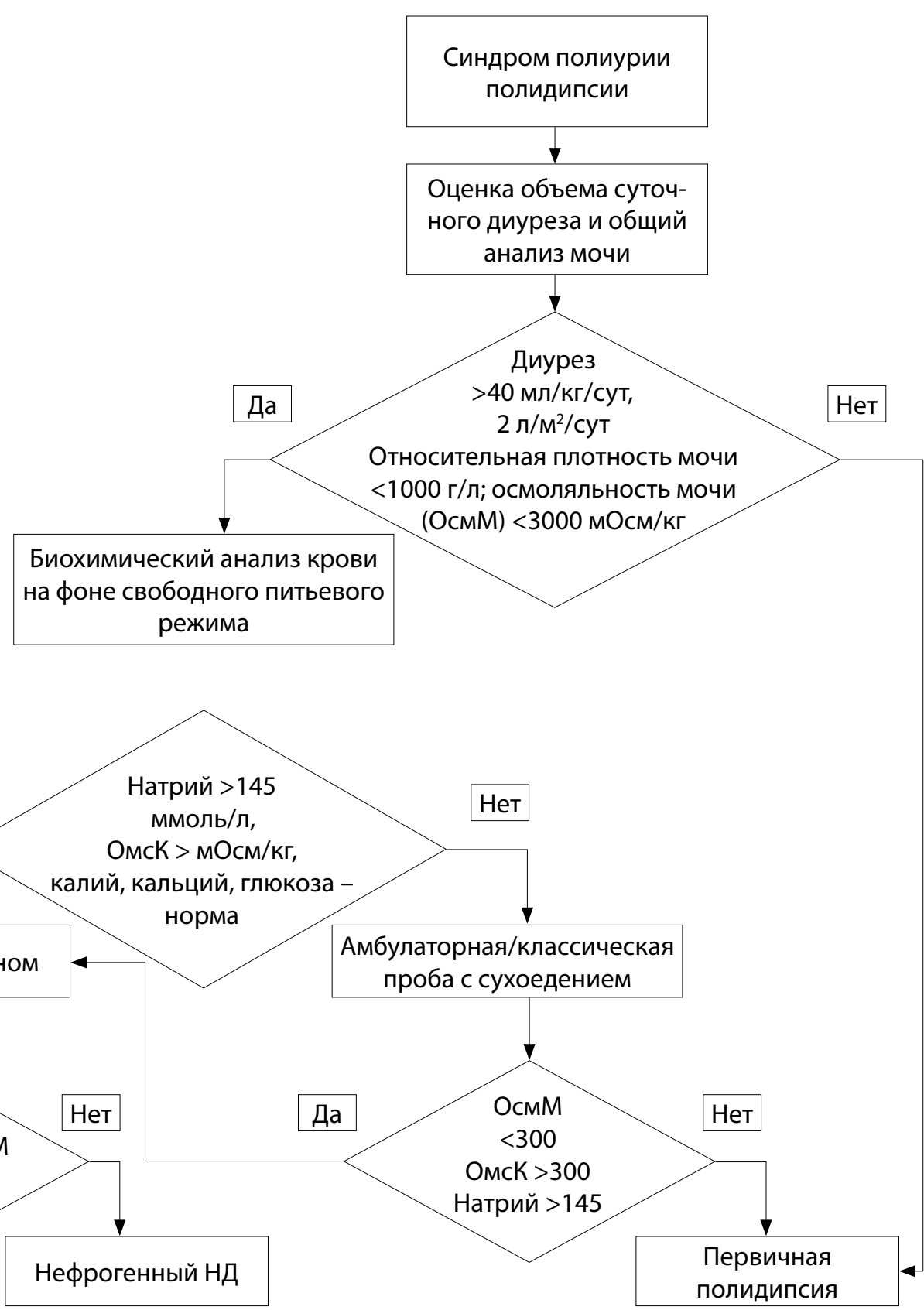

Рис. 3. Алгоритм дифференциальной диагностики несахарного диабета [14].

гипофиза. При подтверждении диагноза «гипофизит» необходима также консультация офтальмолога с осмотром глазного дна и проведением компьютерной периметрии.

Жизненно важно назначение гормональной терапии при утрате гипофизом функций по стимулированию щитовидной железы (вторичный гипотиреоз) и надпочечников (вторичная надпочечниковая недостаточность) (табл. 2). Замещение других утраченных функций важно для восстановления качества жизни.

Тиреоидит является еще одним распространенным нежелательным явлением, которое может протекать с последовательным чередованием двух фаз (рис. 2): тиреотоксической и гипотиреоидной. Все эндокринные АОНЯ характеризуются быстропрогрессирующим течением В сравнении с классическими аутоиммунными заболеваниями эндокринной системы. Такая интенсивность и быстрота нарастания симптомов нередко создают некоторые трудности в ведении пациентов эндокринологами. Так, при выявлении у пациента, получающего онкоиммунотерапию, тиреотоксикоза, важно понимать, что вероятность тиреоидита в сотни раз выше, чем диффузно токсического зоба. При сохранении необходимости дифференциальной диагностики между этими заболеваниями может быть проведена сцинтиграфия щитовидной железы (при отсутствии применения высоких доз йода - например, в виде амиодарона) и/или определение антител к рецептору ТТГ. Назначение препаратов тиреостатиков не показано при тиреоидите, поскольку может спровоцировать усиление цитолитического процесса в ткани щитовидной железы и усугубление тиреотоксикоза с его потенциально опасными кардиальными осложнениями.

Третьим по распространенности эндокринным АОНЯ, по данным исследований, является надпочечниковая недостаточность. К сожалению, в исследованиях на ранних этапах наличие надпочечниковой недостаточности не разделялось четко на вторичную (поражение гипофи- 
Таблица 4. Протоколы функциональных проб у пациентов при подозрении на несахарный диабет

ПРОТОКОЛ ПРОБЫ С СУХОЕДЕНИЕМ (ДЕГИДРАТАЦИОННЫЙ ТЕСТ) ДЛЯ ПОДТВЕРЖДЕНИЯ НЕСАХАРНОГО ДИАБЕТА Исходные действия:

- взять кровь на осмоляльность и натрий;

- собрать мочу для определения объема и осмоляльности;

- взвесить больного;

- измерить АД и пульс.

В дальнейшем через равные промежутки времени в зависимости от состояния больного через 1 или 2 ч повторять эти действия.

Во время пробы больному не разрешается пить, желательно также ограничение пищи (по крайней мере, в течение первых 8 ч проведения пробы); при кормлении пища не должна содержать много воды и легкоусвояемых углеводов, можно употреблять вареные яйца, зерновой хлеб, нежирные сорта мяса, рыбы.

\section{Пробу прекращают:}

- при потере более 3-5\% массы тела;

- невыносимой жажде;

- при объективно тяжелом состоянии пациента;

- повышении натрия и осмоляльности крови выше границ нормы;

- повышении осмоляльности мочи более 650 мОсм/кг.

\section{ПРОВЕДЕНИЕ ПРОБЫ С СУХОЕДЕНИЕМ В АМБУЛАТОРНЫХ УСЛОВИЯХ}

Только для пациентов в стабильном состоянии, с подозрением на наличие полидипсии и выделяющих до 6-8 л мочи в сутки. Цель - получение наиболее концентрированной (последней) порции мочи.

\section{Методика проведения:}

- Попросить больного полностью ограничить прием жидкости в течение того времени, которое он сможет выдержать. Наиболее удобно начало ограничения за несколько часов до сна и во время ночного сна.

- Пациент собирает пробы мочи при возникновении естественной необходимости мочеиспускания ночью и при пробуждении, при этом на анализ приносится только самая последняя порция, поскольку она будет в условиях полного ограничения жидкости являться самой концентрированной.

- В последней порции мочи определяется осмоляльность: показатель, превышающий $650 \mathrm{MOcm/кг,} \mathrm{позволяет}$ исключить любой генез несахарного диабета.

\section{ПРОВЕДЕНИЕ ДЕСМОПРЕССИНОВОГО ТЕСТА (ДЛЯ ДИФФЕРЕНЦИАЛЬНОЙ ДИАГНОСТИКИ ЦЕНТРАЛЬНОЙ} И НЕФРОГЕННОЙ ФОРМ НЕСАХАРНОГО ДИАБЕТА)

Проводится у пациентов после исключения первичной полидипсии, оптимально после пробы с сухоедением.

\section{Методика проведения.}

- Попросить больного полностью опорожнить мочевой пузырь, и в этом анализе мочи определяется ее осмоляльность.

- Ввести десмопрессин: 60 мкг десмопрессина МЕЛТ в виде таблеток подъязычных или 0,1 мг таблетированного десмопрессина под язык до полного рассасывания.

- Пациенту разрешается есть и пить (объем выпиваемой жидкости не должен превышать объем выделенной мочи во время фазы дегидратации).

- Через 2 и 4 ч собрать мочу для определения осмоляльности.

- При центральном НД прирост осмоляльности мочи после приема десмопрессина составляет более 50\%, а при нефрогенном - менее 50\%.

за) или первичную (поражение надпочечников), что не позволяет достоверно определить ее частоту. Представляется важным подчеркнуть, что при подозрении на наличие надпочечниковой недостаточности лечение соответствющими дозами глюкокортикоидов (табл. 2) должно быть начато незамедлительно - сразу после взятия крови на АКТГ и кортизол для последующего подтверждения диагноза и дифференциации уровня поражения [8].

Сахарный диабет является редким эндокринным АОНЯ. Некоторые исследователи считают, что иммунотерапия вызывает новый тип сахарного диабета с острым (фульминантным) началом, который имитирует сахарный диабет 1 типа, но без тех же антител (выявляются у менее 50\% заболевших пациентов), которые обычно обнаруживаются при диагнозе сахарного диабета 1-го типа [9, 10, 11]. Истинное отличие сахарного диабета как осложнения онкоиммунотерапии от сахарного диабета 1 типа в настоящее время невозможно ввиду отсутствия значимого числа хорошо охарактеризованных случаев.

Фульминантное течение заболевания может приводить к несвоевременному распознаванию сахарного диабета, большей частоте манифестирования с такого серьезного осложнения заболевания как кетоацидоз, гипергликемии 20-60 ммоль/л, дегидратации в степени острой почечной недостаточности.

В виде единичных случаев описано возникновение гипопаратиреоза [12] и аутоиммунного центрального несахарного диабета [13]. Оба заболевания могут представлять угрозу для жизни. При гипопаратиреозе -вследствие развития генерализованного судорожного синдрома с вовлечением дыхательных мышц и нарушением дыхания. А при развитии центрального несахарного диабета угроза 
для жизни возникает от обезвоживания при отсутствии свободного доступа к воде и невозможности восполнения потерь жидкости с мочой.

Если лабораторная диагностика первичного гиперпаратиреоза не представляет собой каких-либо сложностей, то дифференциальная диагностика центрального несахарного диабета сопряжена с некоторыми сложностями. Они заключаются в обязательном следовании алгоритму действий (рис. 3) и обязательном проведении функциональных проб (пробы с сухоедением, тест с десмопрессином), протоколы которых представлены в таблице 4. При несоблюдении резко возрастает вероятность развития гипердиагностики заболевания у пациентов с первичной полидипсией (избыточного потребления жидкости), при этом назначение препаратов десмопрессина будет сопровождаться таким грозным нарушением водно-электролитного обмена как водная интоксикация.

В представленной лекции обсуждались только те аутоиммунные эндокринопатии, которые встречались при лечении препаратами ИИКТ. В классической эндокринологии число аутоиммунных эндокринопатий много больше, поэтому не исключено, что с ростом числа пролеченных пациентов онкоиммунотерапевтическими средствами список может расшириться за счет более редко встречающихся эндокринологических АОНЯ.

\section{ЗАКЛЮЧЕНИЕ}

Высокая эффективность онкоиммунотерапии обуславливает расширение области ее применения за счет присоединения различных нозологических форм и количества прошедших данное лечение пациентов. Этот феномен требует большей координации медицинской помощи между специалистами онкологами и врачами других специальностей, в т.ч. эндокринологами, формирования мультидисциплинарных команд по ведению пациентов. Ключевым является обучение эндокринологов и онкологов особенностям лечения, ранней диагностике и коррекции эндокринно-метаболических осложнений.

\section{ДОПОЛНИТЕЛЬНАЯ ИНФОРМАЦИЯ}

Источники финансирования. Поисково-аналитическая работа и подготовка публикации осуществлены в рамках реализации научной программы, поддержанной Российским научным фондом (грант РНФ №17-75-30035).

Конфликт интересов. Авторы декларируют отсутствие явных и потенциальных конфликтов интересов, связанных с публикацией настоящей статьи, о которых следует сообщить.

Участие авторов. Все авторы внесли существенный вклад в проведение поисково-аналитической работы и подготовку статьи, прочли и одобрили финальную версию перед публикацией.

\section{СПИСОК ЛИТЕРАТУРЫ | REFERENCES}

1. Spain L, Diem S, Larkin J. Management of toxicities of immune checkpoint inhibitors. Cancer Treat. Rev. 2016;44:51-60. doi: 10.1016/j.ctrv.2016.02.001

2. Byun DJ, Wolchok JD, Rosenberg LM, Girotra M. Cancer immunotherapy - immune checkpoint blockade and associated endocrinopathies. Nature Reviews Endocrinology. 2017;13(4):195-207. doi: 10.1038/nrendo.2016.205.

3. Zhao C, Tella SH, Del Rivero J, et al. Anti-PD-L1 Treatment Induced Central Diabetes Insipidus. J. Clin. Endocr. Metab. 2018;103(2):365-369. doi: 10.1210/jc.2017-01905

4. Min L. Immune-related endocrine disorders in novel immune checkpoint inhibition therapy. Genes \& Diseases. 2016;3(4):252-256. doi: 10.1016/j.gendis.2016.10.002.

5. Hofmann L, Forschner A, Loquai C, et al. Cutaneous, gastrointestinal, hepatic, endocrine, and renal side-effects of anti-PD-1 therapy. Eur. J. Cancer. 2016;60:190-209. doi: 10.1016/j.ejca.2016.02.025.

6. Weber JS, Kähler KC, Hauschild A. Management of Immune-Related Adverse Events and Kinetics of Response With Ipilimumab. J. Clin. Oncol. 2012;30(21):2691-2697. doi: 10.1200/jco.2012.41.6750.

7. Iwama S, De Remigis A, Callahan MK, et al. Pituitary Expression of CTLA-4 Mediates Hypophysitis Secondary to Administration of CTLA4 Blocking Antibody. Sci. Transl. Med. 2014;6(230):230ra245-230ra245. doi: $10.1126 /$ scitransImed.3008002.

8. Bornstein SR, Allolio B, Arlt W, et al. Diagnosis and Treatment of Primary Adrenal Insufficiency: An Endocrine Society Clinical
Practice Guideline. J. Clin. Endocr. Metab. 2016;101(2):364-389. doi: 10.1210/jc.2015-1710.

9. Tsiogka A, Jansky GL, Bauer JW, Koelblinger P. Fulminant type 1 diabetes after adjuvant ipilimumab therapy in cutaneous melanoma. Melanoma Res. 2017;27(5):524-525. doi: 10.1097/cmr.0000000000000384.

10. Wang Z, Zheng Y, Tu Y, et al. Immunological Aspects of Fulminant Type 1 Diabetes in Chinese. Journal of Immunology Research. 2016;2016:1-6. doi: 10.1155/2016/1858202.

11. Hughes J, Vudattu N, Sznol M, et al. Precipitation of autoimmune diabetes with anti-PD-1 immunotherapy. Diabetes Care. 2015;38(4):e557. doi: $10.2337 / \mathrm{dc} 14-2349$.

12. Win MA, Thein $\mathrm{KZ}$, Qdaisat A, Yeung S-CJ. Acute symptomatic hypocalcemia from immune checkpoint therapy-induced hypoparathyroidism. The American Journal of Emergency Medicine. 2017;35(7):1039. e1035-1039.e1037. doi: 10.1016/j.ajem.2017.02.048.

13. Zhao C, Tella SH, Del Rivero J, et al. Anti-PD-L1 Treatment Induced Central Diabetes Insipidus. J. Clin. Endocr. Metab. 2018;103(2):365-369. doi: 10.1210/jc.2017-01905

14. Дедов И.И., Мельниченко Г.А., Пигарова Е.А., и др. Федеральные клинические рекомендации по диагностике и лечению несахарного диабета у взрослых. // Ожирение и метаболизм. - 2018. T.15. - No. 2 - C. 56-71. [Dedov II, Mel'nichenko GA, Pigarova EA, et al. Federal clinical guidelines on diagnosis and treatment of diabetes insipidus in adults. Obesity and metabolism. 2018;15(2):56-71. (in Russ.)] doi: 10.14341/omet9670.

\section{ИНФОРМАЦИЯ ОБ АВТОРАХ [AUTHORS INFO]}

*Пигарова Екатерина Александровна, к.м.н. [Ekaterina A. Pigarova, MD, PhD]; адрес: 117036, ул. Дмитрия Ульянова, 11 [address: 11 Dm. Ulyanova street, 117036 Moscow, Russia]; ORCID: http://orcid.org/0000-0001-6539-466X; eLibrary SPIN: 6912-6331; e-mail: kpigarova@gmail.com

Дзеранова Лариса Константиновна, д.м.н. [Larisa K. Dzeranova, MD, PhD]; ORCID: http://orcid.org/0000-0002-0327-4619; eLibrary SPIN: 2958-5555; e-mail: dzeranovalk@yandex.ru Нуралиева Нурана Фейзуллаевна, научный сотрудник [Nurana F. Nuralieva, MD]; ORCID: http://orcid.org/0000-0001-6876-3336; eLibrary SPIN-код: 7373-2602; e-mail: dr.nuralievanf@yandex.ru Мельниченко Галина Афанасьевна, д.м.н., профессор, академик PAH [Galina A. Mel`nichenko, MD, PhD, Professor]; ORCID: https://orcid.org/0000-0002-5634-7877; eLibrary SPIN: 8615-0038; e-mail: teofrast2000@mail.ru 


\section{ЦИТИРОВАТЬ:}

Пигарова Е.А., Дзеранова Л.К., Нуралиева Н.Ф., Мельниченко Г.А. Диагностика и лечение эндокринологических осложнений иммунотерапии онкологических заболеваний // Ожирение и метаболизм. — 2018. — Т.15. — №. 3 C. 49-58. doi: $10.14341 /$ omet9834

\section{TO CITE THIS ARTICLE:}

Pigarova EA, Dzeranova LK, Nuralieva NF, Melnichenko GA. Diagnosis and treatment of endocrinological complications of immunotherapy of oncological diseases. Obesity and metabolism. 2018;15(3):49-58. doi: 10.14341/omet9834 